\title{
NONSTANDARD CRITERIA FOR BOREL-MEASURABILITY
}

\author{
D. LANDERS and L. ROGGE
}

\begin{abstract}
Let $(X, \mathscr{A})$ be a measurable space, $(Y, \delta)$ be a metric space with Borel- $\sigma$-algebra $\mathscr{B}$ and $f: X \rightarrow Y$ be a function. If $(Y, \delta)$ is a $\sigma$-compact space, then it is shown that the $\mathscr{A}, \mathscr{B}$-measurability of $f$ is equivalent to the fact that the standard part of ${ }^{*} f$ is constant on $\mathscr{A}$-monads, a result which is not true any more if we replace " $\sigma$-compact" by "locally compact". We moreover prove nonstandard criteria for special classes of $\mathscr{A}, \mathscr{B}$-measurable functions with values in an arbitrary metric space.
\end{abstract}

\section{The results}

In this paper we consider a superstructure containing two given sets $X, Y$ and the set $\mathrm{R}$ of real numbers, and we work with a polysaturated nonstandard model for this superstructure.

Let $\mathscr{A}$ be a $\sigma$-algebra on $X$ and $\mathscr{B}$ be the Borel- $\sigma$-algebra of a metric space $(Y, \delta)$. For $x_{1}, x_{2} \in{ }^{*} X$ we write $x_{1} \approx x_{2}$ iff for all $A \in \mathscr{A}$ there holds: $x_{1} \in{ }^{*} A \Longleftrightarrow x_{2} \in{ }^{*} A$.

According to Ross [5] the following two conditions are equivalent for a function $f: X \rightarrow Y$ :

(I) $f$ is $\mathscr{A}, \mathscr{B}$-measurable.

(II) $x_{1} \underset{\mathscr{A}}{\approx} x_{2} \Rightarrow{ }^{*} f\left(x_{1}\right) \underset{\mathscr{B}}{ }{ }^{*} f\left(x_{2}\right) \quad\left(x_{1}, x_{2} \in{ }^{*} X\right)$.

As the referee has pointed out this equivalence can also be shown with similar methods as those in the proof of Lemma 6.

In ${ }^{*} Y$ we have furthermore an equivalence relation $\approx$ 。 derived from the metric $\delta$, namely $y_{1} \underset{\delta}{\approx} y_{2} \Longleftrightarrow{ }^{*} \delta\left(y_{1}, y_{2}\right) \approx 0$. If $Y=\mathrm{R}$ it is known that for bounded $f$ the $\mathscr{A}, \mathscr{B}$-measurability of $f$ is furthermore equivalent to

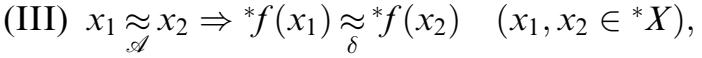

see Ross [5]. This result can also be derived from Loeb [4], Theorem 1.3, p. 67.

Received December 16, 1996; in revised form October 15, 1997. 
The following Theorem 1 extends this result to metric spaces $Y$, and it shows furthermore that condition (III) already implies that $f(X)$ is totally bounded.

1. TheOREM. For a function $f: X \rightarrow Y$ the following conditions are equivalent:

(i) $f$ is $\mathscr{A}, \mathscr{B}$-measurable and $f(X)$ is totally bounded.

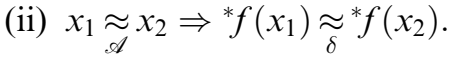

Now we give results which, for the case $Y=\mathrm{R}^{n}$, are nonstandard criteria for the $\mathscr{A}, \mathscr{B}$-measurability of arbitrary not necessarily bounded functions.

Let $\operatorname{fin}\left({ }^{*} Y\right):=\left\{z \in{ }^{*} Y:{ }^{*} \delta\left(z,{ }^{*} y\right)\right.$ is finite for all $\left.y \in Y\right\}$ and $\operatorname{cpt}\left({ }^{*} Y\right):=$ $\cup\left\{{ }^{*} C: C \subset Y\right.$ compact $\}$. Let furthermore $\operatorname{ns}\left({ }^{*} Y\right)$ and $\operatorname{pns}\left({ }^{*} Y\right)$ be the systems of nearstandard-points and prenearstandard-points of ${ }^{*} Y$.

2. TheOREM. For a function $f: X \rightarrow Y$ the following conditions are equivalent:

(i) $f$ is $\mathscr{A}, \mathscr{B}$-measurable and bounded subsets of $f(X)$ are totally bounded.

(ii) $\left(x_{1} \approx \widetilde{\mathscr{A}}_{2} \wedge{ }^{*} f\left(x_{1}\right) \in \operatorname{fin}\left({ }^{*} Y\right)\right) \Rightarrow{ }^{*} f\left(x_{1}\right) \underset{\delta}{*} f\left(x_{2}\right)$.

If $\mathscr{C}$ is a system of subsets of $Y, \sigma(\mathscr{C})$ denotes the smallest $\sigma$-algebra on $Y$ containing $\mathscr{C}$.

3. THEOREM. Let $\mathscr{C}$ be the system of all compact subsets of $Y$. For a function $f: X \rightarrow Y$ the following conditions are equivalent:

(i) $f$ is $\mathscr{A}, \sigma(\mathscr{C})$-measurable.

(ii) $\left(x_{1} \approx \widetilde{\mathscr{A}}_{2} x^{*} f\left(x_{1}\right) \in \operatorname{cpt}\left({ }^{*} Y\right)\right) \Rightarrow{ }^{*} f\left(x_{1}\right) \widetilde{\widetilde{\delta}}^{*} f\left(x_{2}\right)$.

For $\sigma$-compact spaces $Y$, we obtain by Theorem 3 two equivalences for the $\mathscr{A}, \mathscr{B}$-measurability. Both equivalences do not hold for arbitrary metric spaces (see Remark 5).

4. Corollary. Let $(Y, \delta)$ be a $\sigma$-compact metric space. For a function $f: X \rightarrow Y$ the following conditions are equivalent:

(i) $f$ is $\mathscr{A}, \mathscr{B}$-measurable.

(ii) $\left(x_{1} \underset{\mathscr{d}}{\approx} x_{2} \wedge{ }^{*} f\left(x_{1}\right) \in \mathrm{ns}\left({ }^{*} Y\right)\right) \Rightarrow{ }^{*} f\left(x_{1}\right) \widetilde{\widetilde{\delta}}^{*} f\left(x_{2}\right)$.

(iii) $\left(x_{1} \widetilde{\mathscr{A}}_{2} x^{*} f\left(x_{1}\right) \in \operatorname{cpt}\left({ }^{*} Y\right)\right) \Rightarrow{ }^{*} f\left(x_{1}\right) \widetilde{\widetilde{\delta}}^{*} f\left(x_{2}\right)$.

Proof. Direct consequence of $\mathscr{B}=\sigma(\mathscr{C})$, Theorem 3 and (I) $\Longleftrightarrow$ (II).

One can apply Corollary 4 to $Y=\mathrm{R}^{n}$. Observe that in this case fin $\left({ }^{*} \mathrm{R}^{n}\right)=$ $\mathrm{ns}\left({ }^{*} \mathrm{R}^{n}\right)=\operatorname{cpt}\left({ }^{*} \mathrm{R}^{n}\right)$. 
5. Remark. In Corollary 4 condition (i) always implies (ii) and (iii) for arbitrary metric spaces. However, even for locally-compact metric spaces, (ii) (and hence (iii)) do not imply (i): Consider $X:=Y:=\mathrm{R}$ and let $\delta$ be the discrete metric on $\mathbf{R}$. Let $f$ be the identity function on $\mathbf{R}$ and $\mathscr{A}:=\{A \subset \mathrm{R}: A$ or $\mathrm{R} \backslash A$ is countable $\}$. Then $\mathscr{B}=\mathscr{P}(\mathrm{R}), \operatorname{ns}\left({ }^{*} Y\right)=\mathrm{R}$ and $f$ is not $\mathscr{A}, \mathscr{B}$-measurable. However (ii) holds, as $x_{1} \approx \mathscr{A}_{2}$ and ${ }^{*} f\left(x_{1}\right)=x_{1} \in \mathrm{R}$ implies $x_{1}=x_{2}$.

\section{Proof of the Results}

The following Lemma is the crucial tool for the proof of our main results. In the proof of Lemma 6 and Theorem 1 we use that there exists a hyperfinite ${ }^{*} \mathscr{A}$-partition of ${ }^{*} X$, say $\mathrm{P}_{h}$, which refines each finite standard ${ }^{*} \mathscr{A}$-partition (see [4]). Then $x_{1} \approx x_{2}$ if $x_{1}, x_{2} \in E \in \mathrm{P}_{h}$.

6. Lemma. Let $C \subset Y$ be closed. Then $f^{-1}(C) \in \mathscr{A}$ if:

$$
\left(x_{1} \underset{\mathscr{A}}{x_{2}} \wedge^{*} f\left(x_{1}\right) \in{ }^{*} C\right) \Rightarrow{ }^{*} f\left(x_{1}\right) \underset{\delta}{\approx_{\delta}^{*}} f\left(x_{2}\right) .
$$

Proof. Let $n \in \mathrm{N}$. Then, using (C), the existence of $\mathrm{P}_{h}$ and backwards transfer, it follows that there exists a finite $\mathscr{A}$-measurable partition $\left\{E_{1}^{(n)}, \ldots, E_{k(n)}^{(n)}\right\}$ of $X$ with

$$
\left(\forall x_{1}, x_{2} \in E_{i}^{(n)}\right)\left(f\left(x_{1}\right) \in C \Rightarrow \delta\left(f\left(x_{1}\right), f\left(x_{2}\right)\right) \leq 1 / n\right) .
$$

Hence there exists $I_{n} \subset\{1, \ldots, k(n)\}$ with

$$
f^{-1}(C) \subset \cup_{i \in I_{n}} E_{i}^{(n)} \subset\{x \in X: \delta(f(x), C) \leq 1 / n\} .
$$

As $C$ is closed, we have $f^{-1}(C)=\bigcap_{n \in \mathrm{N}}\{x \in X: \delta(f(x), C) \leq 1 / n\}$; since $\cup_{i \in I_{n}} E_{i}^{(n)} \in \mathscr{A}$, we obtain $f^{-1}(C) \in \mathscr{A}$ by $(1)$.

Proof of Theorem 1. (i) $\Rightarrow$ (ii): Let $x_{1} \approx x_{\mathscr{A}} x_{2}$ and $\varepsilon \in \mathrm{R}_{+}$. As $f$ is $\mathscr{A}, \mathscr{B}$ -

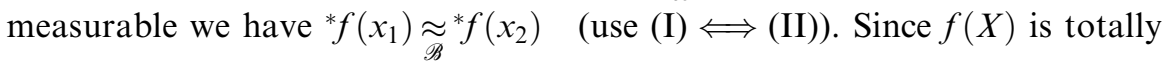
bounded, there exists $y \in Y$ with ${ }^{*} f\left(x_{1}\right) \in{ }^{*}\left(U_{\varepsilon}(y)\right)$, where $U_{\varepsilon}(y)=\{z \in Y$ : $\delta(z, y)<\varepsilon\} \quad$ (see e.g. 24.9(ii) of [3]). As $U_{\varepsilon}(y) \in \mathscr{B}$, we obtain ${ }^{*} f\left(x_{2}\right) \in *^{*}\left(U_{\varepsilon}(y)\right)$. Hence $* f\left(x_{1}\right) \underset{\delta}{*} f\left(x_{2}\right)$.

(ii) $\Rightarrow$ (i): Let $C \subset Y$ be a closed set. Then (ii) implies that condition (C) of Lemma 6 is fulfilled. Hence $f^{-1}(C) \in \mathscr{A}$, whence $f$ is $\mathscr{A}, \mathscr{B}$-measurable. 
Let $\varepsilon \in \mathbf{R}_{+}$. Then, using (ii), the existence of $\mathrm{P}_{h}$ and backwards transfer, it follows that there exists a finite $\mathscr{A}$-measurable partition $\left\{E_{1}, \ldots, E_{k}\right\}$ of $X$ with

$$
\left(x_{1}, x_{2} \in E_{i}\right) \Rightarrow \delta\left(f\left(x_{1}\right), f\left(x_{2}\right)\right) \leq \varepsilon .
$$

Hence $f(X)$ ist totally bounded.

Proof of Theorem 2. (i) $\Rightarrow$ (ii): Let $x_{1} \approx x_{2}$ and ${ }^{*} f\left(x_{1}\right) \in \operatorname{fin}\left({ }^{*} Y\right)$. Then there exists a bounded set $B \subset f(X)$ with ${ }^{*} f\left(x_{1}\right) \in{ }^{*} B$. By (i) $B$ is totally bounded and hence ${ }^{*} f\left(x_{1}\right) \in \operatorname{pns}\left({ }^{*} Y\right)$ (see 24.9(ii) of [3]). Let $\varepsilon \in \mathrm{R}_{+}$. Then there exists $y_{1} \in Y$ with ${ }^{*} f\left(x_{1}\right) \in{ }^{*}\left(U_{\varepsilon}\left(y_{1}\right)\right)$. As $f$ is $\mathscr{A}, \mathscr{B}$-measurable and $x_{1} \underset{\mathscr{A}}{\approx} x_{2}$ we obtain ${ }^{*} f\left(x_{2}\right) \in{ }^{*}\left(U_{\varepsilon}\left(y_{1}\right)\right)$ and hence ${ }^{*} f\left(x_{1}\right) \underset{\delta}{\widetilde{\sigma}^{*}} f\left(x_{2}\right)$.

(ii) $\Rightarrow$ (i): The $\mathscr{A}, \mathscr{B}$-measurability follows from Lemma 6 applied to all bounded and closed sets $C$.

Let $B \subset f(X)$ be a bounded set. It remains to show that $B$ is totally bounded. There exists $X_{0} \subset X$ with $B=f\left(X_{0}\right)$. Let $f_{0}:=f \mid X_{0}$. Then we have for $x_{1}, x_{2} \in{ }^{*} X_{0}$ :

$$
x_{1} \underset{\mathscr{A} \cap X_{0}}{\approx} x_{2} \Rightarrow x_{1} \approx x^{x_{2}} \underset{(\mathrm{ii})}{\Rightarrow} \delta\left({ }^{*} f\left(x_{1}\right),{ }^{*} f\left(x_{2}\right)\right) \approx 0 \Rightarrow{ }^{*} \delta\left({ }^{*} f_{0}\left(x_{1}\right),{ }^{*} f_{0}\left(x_{2}\right)\right) \approx 0 .
$$

Hence by Theorem 1 the set $B=f\left(X_{0}\right)=f_{0}\left(X_{0}\right)$ is totally bounded.

Proof of Theorem 3. (i) $\Rightarrow$ (ii): Let $x_{1} \approx x_{2} x_{2}$ and ${ }^{*} f\left(x_{1}\right) \in{ }^{*} C$ for some $C \in \mathscr{C}$. Since ${ }^{*} C \subset \operatorname{ns}\left({ }^{*} Y\right)$, there exists $y_{1} \in Y$ with ${ }^{*} f\left(x_{1}\right) \widetilde{\widetilde{\delta}}^{*} y_{1}$. Let $\varepsilon \in \mathrm{R}_{+}$ and put $C_{\varepsilon}:=\left\{y \in Y: \delta\left(y, y_{1}\right) \leq \varepsilon\right\}$. Then $C \cap C_{\varepsilon}$ is compact and ${ }^{*} f\left(x_{1}\right) \in{ }^{*}\left(C \cap C_{\varepsilon}\right)$. Since $x_{1} \approx \mathscr{d}^{x_{2}}$ and $f$ is $\mathscr{A}, \sigma(\mathscr{C})$-measurable, we obtain that ${ }^{*} f\left(x_{2}\right) \in{ }^{*}\left(C \cap C_{\varepsilon}\right)$ (use (I) $\Longleftrightarrow($ II) with $\sigma(\mathscr{C})$ instead of $\mathscr{B}$ ). Hence ${ }^{*} \delta\left({ }^{*} f\left(x_{1}\right),{ }^{*} f\left(x_{2}\right)\right) \leq 2 \varepsilon$, whence ${ }^{*} f\left(x_{1}\right) \underset{\delta}{*} f\left(x_{2}\right)$.

(ii) $\Rightarrow$ (i): Apply Lemma 6 to all compact sets $C$.

We thank the referee for improving the presentation. The ideas of the referee led to clearer and much shorter proofs of our main Lemma 6 and Theorem 1 .

\section{REFERENCES}

1. N. Cutland, Nonstandard analysis and its applications, London Math. Soc. Stud. Texts 10 (1988).

2. A. E. Hurd and P. A. Loeb, An Introduction to Nonstandard Real Analysis, Academic Press, Orlando-New York-Tokyo (1985). 
3. D. Landers and L. Rogge, Nichtstandard Analysis, Springer Verlag, Berlin-Heidelberg (1994).

4. P. A. Loeb, A nonstandard representation of measurable spaces, $L_{\infty}$ and $L_{\infty}^{*}$, "Contributions to Nonstandard Analysis" (W. A. J. Luxemburg and A. Robinson, eds.). North-Holland Publ., Amsterdam, (1972), 65-80.

5. D. A. Ross, Measurable transformation in saturated models of analysis, $\mathrm{Ph}$. D. thesis University of Wisconsin (1983).

MATHEMATISCHES INSTITUT DER

UNIVERSITÄT ZU KÖLN

WEYERTAL 86

D-50931 KÖLN

GERMANY

email: landers@mi.uni-koeln.de
FACHBEREICH MATHEMATIK DER

GERHARD-MERCATOR-UNIVERSITÄT GHS DUISBURG

LOTHARSTR. 65

D-47048 DUISBURG

GERMANY

email: rogge@math.uni-duisburg.de 